\title{
39.
}

\section{ON THE DIAMETRAL PLANES OF A SURFACE OF THE SECOND ORDER.}

[From the Cambridge and Dublin Mathematical Journal, vol. I. (1846), pp. 274-278.]

LET $U=A x^{2}+B y^{2}+C z^{2}+2 F y z+2 G x z+2 H x y=0$, be the equation of a surface of the second order referred to its centre, and let $\alpha x+\alpha^{\prime} y+\alpha^{\prime \prime} z=0$ be the equation of one of its diametral planes; then, as usual

$$
\begin{array}{rrr}
(A-u) \alpha+H \alpha^{\prime}+ & G \alpha^{\prime \prime}=0, \\
H \alpha+(B-u) \alpha^{\prime}+ & F \alpha^{\prime \prime} & =0, \\
G \alpha+\quad F \alpha^{\prime}+(C-u) \alpha^{\prime \prime} & =0,
\end{array}
$$

which are equivalent to two independent equations, and consequently capable of determining the ratios $\alpha: \alpha^{\prime}: \alpha^{\prime \prime}$, provided that $u$ satisfy the cubic equation that is obtained by eliminating $\alpha, \alpha^{\prime}, \alpha^{\prime \prime}$ from the three equations.

We have from the second and third, from the third and first, and from the first and second equations respectively,

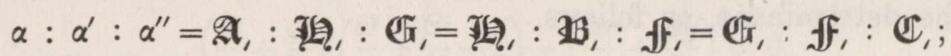

where, if

$$
\begin{aligned}
& \mathfrak{A}=B C-F^{2}, \\
& \mathfrak{a}=C A-G^{2}, \\
& \mathfrak{C}=A B-H^{2}, \\
& \mathfrak{d}=G H-A F, \\
& \mathfrak{G}=H F-B G, \\
& \mathfrak{T}=F G-C H,
\end{aligned}
$$

$\mathfrak{A}_{1}, \mathbb{B}_{1}, \mathfrak{C}_{1}, \mathfrak{d}_{1}, \mathfrak{G}_{1}, \mathfrak{F}_{\mathbf{l}}$, are what these become when $A, B, C$ are changed into 
$A-u, B-u, C-u$, so that

$$
\begin{aligned}
& \mathbf{A},=\mathfrak{A}-(B+C) u+u^{2}, \\
& \boldsymbol{Z} \mathbf{B},=\mathbf{z} \mathbf{b}-(C+A) u+u^{2} \text {, } \\
& \mathfrak{C}_{1}=\mathfrak{C}-(A+B) u+u^{2}, \\
& \sqrt{\mathfrak{F}},=\sqrt{\mathfrak{f}}+F u \text {, } \\
& \boldsymbol{G}_{\mathbf{1}}=\boldsymbol{\varrho} \tilde{\mathrm{x}}+G u \text {, } \\
& \text { 政 }=\text { 积 }+H u \text {. }
\end{aligned}
$$

Hence the equation $a x+\alpha^{\prime} y+\alpha^{\prime \prime} z=0$ may be written in the three forms

$$
\begin{aligned}
& \mathfrak{A}, x+\mathfrak{I}, y+\mathfrak{G}_{1}, z=0 \text {, } \\
& \text { 殖, } x+\mathfrak{B}, y+\sqrt{\mathfrak{f}}, z=0 \text {, } \\
& \mathbb{G}, x+\sqrt{f}, y+\mathfrak{C}, z=0 \text {; }
\end{aligned}
$$

or, what comes to the same thing, as follows,

$$
\begin{aligned}
& \mathbf{A} x+\text { 㯊 } y+\mathbf{G} z+u(A x+H y+G z)+v x=0 \text {, } \\
& \text { 政 } x+\mathfrak{B} y+\sqrt{\mathfrak{f} z}+u(H x+B y+F z)+v y=0 \text {, } \\
& \mathfrak{C} x+\sqrt{\mathfrak{F}} y+\mathfrak{C} z+u(G x+F y+C z)+v z=0 \text {, }
\end{aligned}
$$

in which for shortness $v$ has been written instead of

$$
u^{2}-(A+B+C) u \text {. }
$$

The elimination of $u, v$ from these equations gives a result $\Theta=0$, where $\Theta$ is a homogeneous function of the third order in $x, y, z$; and this equation, it is evident, must belong to the three diametral planes jointly, i.e. $\Theta$ must be the product of three linear factors, each of which equated to zero would correspond to a diametral plane. Thus the system of diametral planes is given by

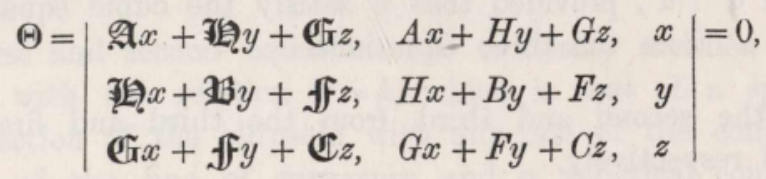

or developing the determinant, as follows,

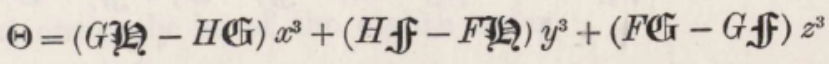

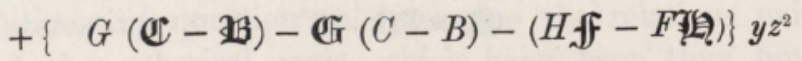

$$
\begin{aligned}
& +\left\{H(\mathfrak{A}-\mathfrak{C})-\mathfrak{I}\left(A-C_{1}-(F \mathfrak{G}-G \sqrt{\mathfrak{F}})\right\} z x^{2}\right. \\
& +\{F(\boldsymbol{B}-\mathbf{A})-\sqrt{\mathfrak{f}}(B-A)-(G \mathfrak{F}-H \tilde{G})\} x y^{2} \\
& +\{-H(\mathbb{C}-\boldsymbol{B})+\mathfrak{Z}(C-B)+(F \mathbb{G}-G \sqrt{\mathfrak{F}})\} y^{2} z \\
& +\{-F(\mathfrak{A}-\mathfrak{C})+\sqrt{f}(A-C)+(G \mathbb{J}-H \in \mathbb{G})\} z^{2} x \\
& +\left\{-G(\mathfrak{B}-\mathfrak{A})+\mathfrak{C}(B-A)+\left(H \sqrt{\mathfrak{F}}-F^{2}\left[\begin{array}{l}
\mid \\
-
\end{array}\right)\right\} x^{2} y\right. \\
& +(C \mathfrak{B}-B \mathfrak{A}+\mathfrak{A C}-C \mathfrak{A}+B \mathfrak{A}-A \mathfrak{A}) x y z ;
\end{aligned}
$$


or reducing

$$
\begin{aligned}
\Theta= & \left\{F\left(G^{2}-H^{2}\right)-G H(C-B)\right\} x^{3} \\
& +\left\{G\left(H^{2}-F^{2}\right)-H F(A-C)\right\} y^{3} \\
& +\left\{H\left(F^{2}-G^{2}\right)-F G(B-A)\right\} z^{3} \\
& +\left\{G(A-B)(B-C)+F H(A+B-2 C)+G\left(F^{2}+G^{2}-2 H^{2}\right)\right\} y z^{2} \\
& +\left\{H(B-C)(C-A)+G F(B+C-2 A)+H\left(G^{2}+H^{2}-2 F^{2}\right)\right\} z x^{2} \\
& +\left\{F(C-A)(A-B)+G H(C+A-2 B)+F\left(H^{2}+F^{2}-2 G^{2}\right)\right\} x y^{2} \\
& +\left\{H(B-C)(C-A)+F G(C+A-2 B)+H\left(H^{2}+F^{2}-2 G^{2}\right)\right\} y^{2} z \\
& +\left\{F(C-A)(A-B)+G H(A+B-2 C)+F\left(F^{2}+G^{2}-2 H^{2}\right)\right\} z^{2} x \\
& +\left\{G(A-B)(B-C)+H F(B+C-2 A)+G\left(G^{2}+H^{2}-2 F^{2}\right)\right\} x^{2} y \\
& -\left\{(A-B)(B-C)(C-A)+(B-C) F^{2}+(C-A) G^{2}+(A-B) H^{2}\right\} x y z .
\end{aligned}
$$

In the case of curves of the second order, the result is much more simple; we have

$$
\begin{gathered}
\Theta=\left|\begin{array}{cc}
A x+H y, & x \\
H x+B y, & y
\end{array}\right|=0, \\
\Theta=H\left(y^{2}-x^{2}\right)+(A-B) x y=0,
\end{gathered}
$$

i.e.

for the equation of the two diameters.

The above formulæ may be applied to the question of finding the diametral planes of the cone circumscribed about a given surface of the second order, (or of the lines bisecting the angles made by two tangents of a curve of the second order). Considering the latter question first: if

$$
\frac{x^{2}}{a^{2}}+\frac{y^{2}}{b^{2}}-1=0
$$

be the equation of the curve, and $\alpha, \beta$ the coordinates of the point of intersection of the two tangents, the equation of the pair of tangents is

$$
\left(\frac{x^{2}}{a^{2}}+\frac{y^{2}}{b^{2}}-1\right)\left(\frac{a^{2}}{a^{2}}+\frac{\beta^{2}}{b^{2}}-1\right)-\left(\frac{\alpha x}{a^{2}}+\frac{\beta y}{b^{2}}-1\right)^{2}=0 ;
$$

or making the point of intersection the origin,

$$
\left(\frac{x^{2}}{a^{2}}+\frac{y^{2}}{b^{2}}\right)\left(\frac{\alpha^{2}}{a^{2}}+\frac{\beta^{2}}{b^{2}}-1\right)-\left(\frac{a x}{a^{2}}+\frac{\beta y}{b^{2}}\right)^{2}=0
$$

i.e.

$$
(\beta x-\alpha y)^{2}-\left(b^{2} x^{2}+a^{2} y^{2}\right)=0 ;
$$

whence $A=\beta^{2}-b^{2}, B=\alpha^{2}-a^{2}, H=-\alpha \beta$, and the equation to the lines bisecting the angles formed by the tangents is

$$
\alpha \beta\left(x^{2}-y^{2}\right)-\left\{\alpha^{2}-\beta^{2}-\left(a^{2}-b^{2}\right)\right\} x y=0,
$$

which is the same for all confocal ellipses; whence the known theorem,

"If there be two confocal ellipses, and tangents be drawn to the second from any point $P$ of the first, the tangent and normal of the first conic at the point $P$, bisect the angles formed by the two tangents in question."

c. 
In the case of surfaces, the equation of the circumscribing cone referred to its vertex as origin, is

$$
\left(\frac{x^{3}}{a^{2}}+\frac{y^{2}}{b^{2}}+\frac{z^{2}}{c^{2}}\right)\left(\frac{a^{2}}{a^{2}}+\frac{\beta^{2}}{b^{2}}+\frac{\gamma^{2}}{c^{2}}-1\right)-\left(\frac{a x}{a^{2}}+\frac{\beta y}{b^{2}}+\frac{\gamma z}{c^{2}}\right)^{2}=0
$$

whence

$$
\begin{aligned}
& A=\beta^{2} c^{2}+\gamma^{2} b^{2}-b^{2} c^{2}, \\
& B=\gamma^{2} a^{2}+a^{2} c^{2}-a^{2} c^{2}, \\
& C=\alpha^{2} b^{2}+\beta^{2} a^{2}-b^{2} a^{2}, \\
& F=-a^{2} \beta \gamma, \\
& G=-b^{2} \gamma \alpha, \\
& H=-c^{2} \alpha \beta .
\end{aligned}
$$

Hence, omitting the factor $b^{2} c^{2} \alpha^{2}+c^{2} a^{2} \beta^{2}+a^{2} b^{2} \gamma^{2}-a^{2} b^{2} c^{2}$, we have

$$
\begin{aligned}
& \mathfrak{A}=a^{2}-a^{2}, \\
& \mathfrak{B}=\beta^{2}-b^{2}, \\
& \mathfrak{C}=\gamma^{2}-c^{2}, \\
& \mathbb{f}=\beta \gamma, \\
& \mathbb{G}=\gamma \alpha, \\
& \mathfrak{G}=\alpha \beta ;
\end{aligned}
$$

and the equation of the system of diametral planes becomes

$$
\begin{aligned}
\Theta=0= & \alpha^{2} \beta \gamma\left(c^{2}-b^{2}\right) x^{3}+\beta^{2} \gamma \alpha\left(a^{2}-c^{2}\right) y^{3}+\gamma^{2} \alpha \beta\left(b^{2}-a^{2}\right) z^{3} \\
+ & \gamma \alpha\left\{\alpha^{2}\left(c^{2}-b^{2}\right)+\beta^{2}\left(b^{2}+c^{2}-2 a^{2}\right)-\gamma^{2}\left(b^{2}-a^{2}\right)+\left(b^{2}-a^{2}\right)\left(c^{2}-b^{2}\right)\right\} y z^{2} \\
+ & \alpha \beta\left\{-a^{2}\left(c^{2}-b^{2}\right)+\beta^{2}\left(a^{2}-c^{2}\right)+\gamma^{2}\left(c^{2}+a^{2}-2 b^{2}\right)+\left(c^{2}-b^{2}\right)\left(a^{2}-c^{2}\right)\right\} z x^{2} \\
+ & \gamma \alpha\left\{a^{2}\left(a^{2}+b^{2}-2 c^{2}\right)-\beta^{2}\left(a^{2}-c^{2}\right)+\gamma^{2}\left(b^{2}-a^{2}\right)+\left(a^{2}-c^{2}\right)\left(b^{2}-a^{2}\right)\right\} x y^{2} \\
& -\alpha \beta\left\{\alpha^{2}\left(c^{2}-b^{2}\right)-\beta^{2}\left(a^{2}-c^{2}\right)-\gamma^{2}\left(b^{2}+c^{2}-2 a^{2}\right)-\left(a^{2}-c^{2}\right)\left(c^{2}-b^{2}\right)\right\} y^{2} z \\
- & \beta \gamma\left\{-a^{2}\left(c^{2}+a^{2}-2 b^{2}\right)+\beta^{2}\left(a^{2}-c^{2}\right)-\gamma^{2}\left(b^{2}-a^{2}\right)-\left(b^{2}-a^{2}\right)\left(a^{2}-c^{2}\right)\right\} z^{2} x \\
- & \gamma \alpha\left\{-\alpha^{2}\left(c^{2}-b^{2}\right)-\beta^{2}\left(a^{2}+b^{2}-2 c^{2}\right)+\gamma^{2}\left(b^{2}-a^{2}\right)-\left(c^{2}-b^{2}\right)\left(b^{2}-a^{2}\right)\right\} x^{2} y \\
+ & \left\{\left(a^{2}-b^{2}\right)\left(b^{2}-c^{2}\right)\left(c^{2}-a^{2}\right)+\right. \\
& \left(\alpha^{4}+\beta^{2} \gamma^{2}\right)\left(b^{2}-c^{2}\right)-\left(\beta^{4}+\gamma^{2} \alpha^{2}\right)\left(c^{2}-a^{2}\right)-\left(\gamma^{4}+a^{2} \beta^{2}\right)\left(a^{2}-b^{2}\right)+ \\
& \left.\alpha^{2}\left(b^{2}-c^{2}\right)\left(2 a^{2}-b^{2}-c^{2}\right)+\beta^{2}\left(c^{2}-a^{2}\right)\left(2 b^{2}-c^{2}-a^{2}\right)+\gamma^{2}\left(a^{2}-b^{2}\right)\left(2 c^{2}-a^{2}-b^{2}\right)\right\} x y z ;
\end{aligned}
$$

and since this is a function of $a^{2}-b^{2}, b^{2}-c^{2}$, and $c^{2}-a^{2}$, the equation is the same for all confocal ellipsoids; whence the known theorem, "The axes of the circumscribing cone having its vertex in a given point $P$, are tangents to the curves of intersection of the three surfaces, confocal with the given surface, which pass through the point $P$." 